Vol. 38 (1988) [345-349]

\title{
COMPLEX VECTOR BUNDLES ON REAL ALGEBRAIC VARIETIES OF SMALL DIMENSION
}

\author{
WOJCIECH KUCHARZ
}

\begin{abstract}
Let $X$ be an affine real algebraic variety. In this paper, assuming that $\operatorname{dim} X \leqslant 7$ and that $X$ satisfies some other reasonable conditions, we give a characterisation of those continuous complex vector bundles on $X$ which are topologically isomorphic to algebraic complex vector bundles on $X$.
\end{abstract}

\section{INTRODUCTION}

Let $F$ denote one of the fields $\mathbf{R}, \mathbb{C}$ or $\mathbf{H}$ (the reals, complexes or quaternions). Let $X$ be an affine real algebraic variety (that is, $X$ is biregularly isomorphic to an algebraic subset of $\mathbf{R}^{n}$ for some $n$; for definitions and notions of real algebraic geometry we refer to the book [2]). Denote by $A$ the ring of $\mathrm{R}$-valued regular functions on $X$ and set $A(F)=A \otimes_{\mathbf{R}} F$. We shall consider $A(F)$ as a subring of the ring $B(F)$ of continuous $F$-valued functions on $X$. A continuous $F$-vector bundle $\xi$ on $X$ is said to admit an algebraic structure if there exists a finitely generated projective $A(F)$ module $P$ such that the $F$-vector bundle on $X$ associated, in the usual way (see [17]), with $P \otimes_{A(F)} B(F)$ is topologically isomorphic to $\xi$ (an equivalent, more geometric, definition is given in [2] and [1]).

The following problem has attracted the attention of several mathematicians.

Problem. Characterise continuous $F$-vector bundles on $X$ admitting an algebraic structure.

Until very recently, despite considerable effort, the situation was well understood, only in a few special cases (see $[8,10,11]$ and $[16]$ for a short survey). For $\operatorname{dim} X \leqslant 3$ and $F=\mathbf{R}$ a very satisfactory solution is given in $[4]$ (see also $[3,12,13]$ for earlier results). In [1] (see also [7]) most results are first obtained for $C$-vector bundles and then many of them are extended on to $F$-vector bundles, $F=\mathrm{R}$ or $\mathrm{H}$, by using the realification and quaternionification. The main tool of $[1]$, which will also be used here, is the functor $H_{\mathrm{C}-\mathrm{alg}}^{\text {even }}(\cdot, \mathrm{Z})$ from affine real algebraic varieties to graded rings (we recall the definition of $H_{\mathrm{C}-\text { alg }}^{\text {even }}(\cdot, Z)$ in the next section). If $X$ is an affine real algebraic

Received 5 January 1988

Research supported by NSF Grant DMS-8602672.

Copyright Clearance Centre, Inc. Serial-fee code: 0004-9729/88 \$A2.00+0.00. 
variety, then $H_{C-a \lg }^{\text {even }}(X, Z)$ is a subring of the cohomology ring $H^{\text {even }}(X, Z)$. It is known that the total Chern class $c(\xi)$ of a given continuous $C$-vector bundle $\xi$ on $X$ belongs to $H_{\mathrm{C}-\text { alg }}^{\text {even }}(X, Z)$ if $\xi$ admits an algebraic structure [1] (see also [7]). In this paper we show that if $c(\xi)$ is in $H_{\mathrm{C}-\mathrm{alg}}^{\text {even }}(X, \mathbf{Z}), \operatorname{dim} X \leqslant 7$ and $X$ satisfies some reasonable extra conditions, then $\xi$ admits an algebraic structure. This result has been announced in [7], for $\operatorname{dim} X \leqslant 5$, but no proof is given in the detailed version [1] of [7]. It should be mentiioned that the ring $H_{\mathrm{C}-\mathrm{alg}}^{\text {even }}(X, Z)$ is computed in [1] (see also [7]) for a large class of varieties $X$. It turns out that, in many cases, $H_{\mathrm{C}-\mathrm{alg}}^{\text {even }}(X, Z)$ is small as compared with $H^{\text {even }}(X, Z)$. This imposes strong restrictions on continuous $\mathbb{C}$-vector bundles on $X$ admitting an algebraic structure (see also [5] for other applications of $\left.H_{\mathrm{C}-\mathrm{alg}}^{\text {even }}(X, \mathrm{Z})\right)$.

\section{THE RESULT}

For simplicity we shall recall the definition of $H_{C-a l g}^{\text {even }}(X, Z)$ only for nonsingular affine real algebraic varieties $X$ (see [1] for the general case), which will be sufficient for our purposes.

Let $V$ be a quasi-projective nonsingular $n$-dimensional complex algebraic variety. One defines the natural homomorphism

$$
c l: A^{*}(V) \rightarrow H^{*}(V, \mathrm{Z}),
$$

where $A^{*}(V)$ is the Chow ring of $V$ and $H^{*}(V, Z)$ is the Cech cohomology of $V$, as follows. Let $Y \subseteq V$ be a closed irreducible subvariety of dimension $k$. Let $\{Y\}$ be the element of $A^{n-k}(V)$ represented by $Y$ and let $[Y]$ be the fundamental class of $Y$ in the Borel-Moore homology group $H_{2 k}^{B M}(Y, Z)$ (see [6] or [0, Chapter 19]). Then $\operatorname{cl}(\{Y\})$ is the element of $H^{2 n-2 k}(V, Z)$ which corresponds, via Poincaré duality, to the image of $[Y]$ in $H_{2 k}^{B M}(V, Z)$ under the homomorphism $H_{2 k}^{B M}(Y, Z) \rightarrow H_{2 k}^{B M}(V, Z)$ induced by the inclusion $Y \subseteq V$. Extending by linearity, $c l$ defines a natural homomorphism $c l: A^{*}(V) \rightarrow H^{*}(V, \mathrm{Z})$. Clearly, the image of $c l$ is contained in $H^{\text {even }}(V, \mathrm{Z})$. We set

$$
H_{\mathrm{alg}}^{\mathrm{even}}(V, \mathbf{Z})=\operatorname{cl}\left(A^{*}(V)\right)
$$

Now let $X$ be an affine real algebraic variety (any such variety can be embedded as a locally closed algebraic subvariety in some real projective space $R P^{n}$ ). Consider $\mathbf{R} P^{n}$ as a subset of the complex projective space $C P^{n}$ and suppose for a moment that $X$ is embedded in $R P^{n}$ as a locally closed subvariety. Moreover, assume that $X$ is nonsingular. Let $U$ be a Zariski neighbourhood of $X$ in the set of nonsingular points of the Zariski (complex) closure of $X$ in $C P^{n}$. We define $H_{\mathrm{C}-\mathrm{alg}}^{\mathrm{even}}(X, Z)$ by

$$
H_{\mathrm{C}-\mathrm{alg}}^{\mathrm{even}}(X, \mathbf{Z})=H^{*}\left(i_{U}\right)\left(H_{\mathrm{alg}}^{\mathrm{even}}(U, \mathbf{Z})\right) \text {, }
$$


where $i_{U}: X \rightarrow U$ is the inclusion mapping. One easily sees that $H_{\mathrm{C}-\text { alg }}^{\text {even }}(X, Z)$ does not depend on the choice of the embedding of $X$ in $R P^{n}$ and the choice of $U$ (see [1, Section 3]).

Theorem. Let $X$ be an affine nonsingular real algebraic variety and let $\xi$ be a continuous $\mathrm{C}$-vector bundle of constant rank on $X$. Assume that $X$ is compact, $\operatorname{dim} X \leqslant 7$, and the groups $H^{6}(X, Z)$ and $H^{6}(X, Z) / H_{\mathrm{C}-\mathrm{alg}}^{6}(X, Z)$ have no 2-torsion. Then the following conditions are equivalent:

(a) $\xi$ admits an algebraic structure;

(b) the total Chern class $c(\xi)$ of $\xi$ belongs to $H_{\mathrm{C}-\mathrm{alg}}^{\text {even }}(X, \mathrm{Z})$.

Proof: The implication (a) $\Longrightarrow(b)$ is proved in [1] (see also [7]) for all affine real algebraic varieties $X$ without any additional restrictions.

Before beginning the proof of $(b) \Longrightarrow(a)$, it will be convenient to collect a few facts.

Lemma. Let $X$ be a locally closed real algebraic subvariety of $\mathrm{R} P^{n}$ and let $V$ be a Zariski neighbourhood of $X$ in the Zariski (complex) closure of $X$ in $C P^{n}$. Let $\eta$ be an algebraic vector bundle on $V$. Then:

(i) there exists an affine open complex subvariety $U$ of $V$ containing $X$;

(ii) the restriction $\eta \mid X$ of $\eta$ to $X$, considered as a continuous $C$-vector bundle on $X$, admits an algebraic structure;

(iii) if $V$ is nonsingular, then $c l(C(\eta))=c(\eta)$, where $C(\eta)$ and $c(\eta)$ are the total Chern classes of $\eta$ with values in $A^{*}(V)$ and $H^{\text {even }}(V, \mathrm{Z})$, respectively.

Proof: (i) and (ii) are completely elementary (see for example [1, Proposition 5.1]), while (iii) is proved in [6].

Now we can return to the proof of $(b) \Longrightarrow(a)$. We may assume that $X$ is a locally closed subvariety of $\mathbf{R} P^{n}$. Let $U$ be an affine Zariski neighbourhood of $X$ in the set of nonsingular points of the Zariski closure of $X$ in $C P^{n}$ (see (i) of the Lemma) and let

$$
r: H^{*}(U, \mathbf{Z}) \rightarrow H^{*}(X, \mathbf{Z})
$$

be the homomorphism induced by the inclusion $X \subseteq U$. We may assume that for each $i=1,2,3$, there exists an element $a_{i}$ in $A^{i}(U)$ such that

$$
r\left(c l\left(a_{i}\right)\right)=c_{i}(\xi) .
$$

Let $\eta_{1}$ and $\eta_{2}$ be algebraic vector bundles on $U$ satisfying rank $\eta_{1}=1, C_{1}\left(\eta_{1}\right)=a_{1}$, $C_{1}\left(\eta_{2}\right)=0$ and $C_{2}\left(\eta_{2}\right)=a_{2}$ (the existence of $\eta_{1}$ is obvious, while the existence of $\eta_{2}$ follows at once from the Grothendieck formula [9, Example 15.3.6]). 
Let

$$
\rho: H^{*}(X, \mathrm{Z}) \rightarrow H^{*}(X, \mathrm{Z} / 2)
$$

be the reduction (modulo 2) homomorphism. It follows from the $\mathrm{Wu}$ formula $[14, \mathrm{p}$. 94], applied to $\xi$ and $\eta_{2} \mid X$, that

$$
\begin{aligned}
S q^{2}\left(\rho\left(c_{2}(\xi)\right)\right) & =\rho\left(c_{1}(\xi) c_{2}(\xi)-c_{3}(\xi)\right) \\
S q^{2}\left(\rho\left(c_{2}\left(\eta_{2} \mid X\right)\right)\right) & =\rho\left(c_{3}\left(\eta_{2} \mid X\right)\right),
\end{aligned}
$$

where $S q^{2}: H^{4}(X, Z / 2) \rightarrow H^{6}(X, Z / 2)$ is the Steenrod square (to obtain the second equality, one uses $C_{1}\left(\eta_{2}\right)=0$ and condition (iii) of the Lemma, which guarantees that $c_{1}\left(\eta_{2}\right)=0$ and hence $\left.c_{1}\left(\eta_{2} \mid X\right)=0\right)$.

Let $a=a_{1} a_{2}-a_{3}+C_{3}\left(\eta_{2}\right)$. Then, by (1), (2) and condition (iii) of the Lemma,

$$
\begin{aligned}
\rho(r(c l(a))) & =\rho\left(r\left(c l\left(a_{1} a_{2}-a_{3}\right)\right)\right)+\rho\left(r\left(c l\left(C_{3}\left(\eta_{2}\right)\right)\right)\right) \\
& =\rho\left(c_{1}(\xi) c_{2}(\xi)-c_{3}(\xi)\right)+\rho\left(c_{3}\left(\eta_{2} \mid X\right)\right) \\
& =S q^{2}\left(\rho\left(c_{2}(\xi)\right)\right)+S q^{2}\left(\rho\left(c_{2}\left(\eta_{2} \mid X\right)\right)\right) \\
& =S q^{2}\left(\rho\left(c_{2}(\xi)\right)\right)+S q^{2}\left(\rho\left(c_{2}(\xi)\right)\right) \\
& =0 .
\end{aligned}
$$

Hence $r(c l(a))=-2 \nu$ for some $\nu$ in $H^{6}(X, Z)$. Since $2 \nu$ is in $H_{\mathbb{C}-\text { alg }}^{6}(X, Z)$ and the group $H^{6}(X, Z) / H_{C-a l g}^{6}(X, Z)$ has no 2-torsion, it follows that $\nu$ is in $H_{\mathbb{C}-a l g}^{6}(X, Z)$. Shrinking $U$, if necessary, we may assume that $\nu=r(c l(b))$ for some $b$ in $A^{3}(U)$. By the Grothendieck formula [9, Example 15.3.6], there exists a vector bundle $\eta_{3}$ on $U$ such that $C_{i}\left(\eta_{3}\right)=0$ for $i=1,2$ and $C_{3}\left(\eta_{3}\right)=2 b$. Let $\eta=\eta_{1} \oplus \eta_{2} \oplus \eta_{3}$. Then

$$
\begin{aligned}
& C_{i}(\eta)=a_{i} \text { for } i=1,2 \\
& C_{3}(\eta)=a_{1} a_{2}+C_{3}\left(\eta_{2}\right)+2 b
\end{aligned}
$$

Hence, using (1), we obtain

$$
\begin{aligned}
c_{i}(\eta \mid X) & =r\left(c l\left(a_{i}\right)\right)=C_{i}(\xi) \text { for } i=1,2 \\
c_{3}(\eta \mid X) & =r\left(\operatorname{cl}\left(a_{1} a_{2}+C_{3}\left(\eta_{2}\right)+2 b\right)\right) \\
& =r\left(\operatorname{cl}\left(a_{1} a_{2}+C_{3}\left(\eta_{2}\right)\right)+2 r(\operatorname{cl}(b))\right) \\
& =r\left(\operatorname{cl}\left(a+a_{3}\right)\right)-r(\operatorname{cl}(a)) \\
& =r\left(\operatorname{cl}\left(a_{3}\right)\right) \\
& =c_{3}(\xi) .
\end{aligned}
$$

Thus $c(\xi)=c(\eta \mid X)$ and, by Peterson's theorem [15], $\xi$ and $\eta \mid X$ are stably equivalent (here we use the assumptions that $\xi$ is of constant rank and $H^{6}(X, \mathrm{Z})$ has no 
2-torsion). Moreover, by condition (ii) of the Lemma, $\eta \mid X$ admits an algebraic structure. It is well-known (see [18, Theorem $2.2(\mathrm{a})]$ or [2, Chapter 12]) that a continuous vector bundle on a compact affine real algebraic variety admits an algebraic structure if and only if it is stably equivalent to a vector bundle admitting an algebraic structure. Thus $\xi$ admits an algebraic structure.

\section{REFERENCES}

[1] J. Bochnak, M. Buchuer and W. Kucharz, 'Vector bundles over real algebraic varieties', (preprint) (University of New Mexico).

[2] J. Bochnak, M. Coste and M.-F. Roy, Géométrie Algébrique Réelle (Erge. Math. Grenzgeb (3) 12 Springer, 1987).

[3] J. Bochnak and W. Kucharz, 'Sur les classes d'homologie représentables par des hypersurfaces algébriques réelles', C. R. Acad. Sci. Paris Sér. I Math. 305 (1987), 609-611.

[4] J. Bochnak and W. Kucharz, 'On homology classes represented by real algebraic hypersurfaces', (preprint) (University of New Mexico).

[5] J. Bochnak and W. Kucharz, 'Morphismes algébriques réelles à valeurs dans $S^{2 k}$ et $K$-theorie algébrique', C. R. Acad. Sci. Paris Sér. I Math. 305 (1987), 545-547.

[6] A. Borel and A. Haefliger, 'La classe d'homologie fondamental d'un espace analy tique', Bull. Soc. Math. France 89 (1961), 461-513.

[7] M. Buchner and W. Kucharz, 'Algebraic vector bundles over real algebraic varieties', Bull. Amer. Math. Soc. 17 (1987), 279-282.

[8] R. Fossum, 'Vector bundles over spheres are algebraic', Invent. Math. 8 (1969), 222-225.

[0] W. Fulton, Intersection Theory 2 (Erge. Math. Grenzgeb (3) Springer, 1984).

[10] A.V. Geramita and L. G. Roberts, 'Algebraic vector bundles on projective spaces', Invent. Math. 10 (1970), 298-304.

[11] J.-P. Jouanolou, 'Comparison des $K$-théories algebrique et topologique de quelque variétés algébrique', C. R. Acad. Sci. Paris, Sér A, Ser. A 272 (1971), 1373-1375.

[12] W. Kucharz, 'Vector bundles over real algebraic surfaces and threefolds', Compositio Math. 60 (1986), 209-225.

[13] W. Kucharz, 'Topology of real algebraic threefolds', Duke Math. J. 53 (1986), 1073-1079.

[14] J. Milnor and J. Stasheff, Characteristic Classes (Princeton Univ. Press, Princeton, 1974).

[15] F.P. Peterson, 'Some remarks on Chern classes', Ann. of Math. (2) 60 (1959), 414-420.

[10] L.G. Roberts, 'Comparison of algebraic and topological K-theory', Algebraic K.Theory II. Lecture Notes in Math. 342, pp. 74-78 (Springer-Verlag, Berlin and New York, 1973).

[17] R.G. Swan, 'Vector bundles and projective modules', Trans. Amer. Math. Soc. 105 (1962), 264-277.

[18] R.G. Swan, 'Topological examples of projective modules', Trans. Amer. Math. Soc. 230 (1977), 201-234.

Department of Mathematics and Statistics

University of New Mexico

Albuquerque, New Mexico 87131

United States of America. 